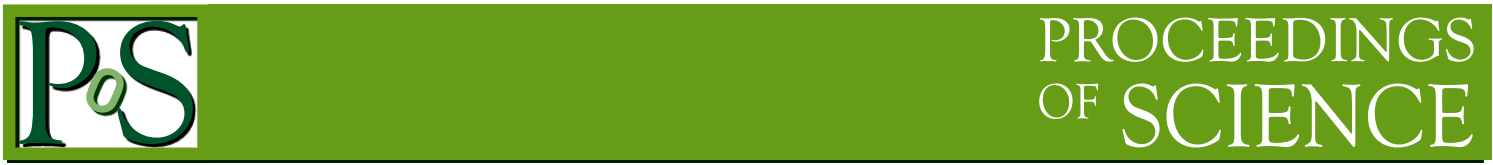

\title{
Higgs and BSM physics at CLIC
}

\section{Gordana Milutinovic-Dumbelovic ${ }^{1}$}

On behalf of the CLICdp collaboration

Institute of Nuclear Sciences Vinca

Mike Petrovica Alasa 12-14, 11000 Belgrade, Serbia

E-mail: gordanamdevinca.rs

The Compact Linear Collider (CLIC) is an option for a future electron-positron collider operating at centre-of-mass energies from a few hundred $\mathrm{GeV}$ up to $3 \mathrm{TeV}$. This paper discusses the Higgs and BSM physics reach of CLIC operating in several energy stages. The presented results are based on physics benchmark analyses using full detector simulations. The initial stage of operation near the top quark pair production threshold allows to study Higgs boson production in the Higgsstrahlung and WW-fusion processes, resulting in model-independent determinations of the Higgs couplings. High-energy operation, here assumed at 1.4 and $3 \mathrm{TeV}$, gives access to rarer Higgs decays and production processes such as double Higgs production, which is sensitive to the Higgs self-coupling. In the second part of the paper, examples for direct and indirect new physics searches are given. In both cases, the achievable sensitivities generally rise with the centre-ofmass energy.

The European Physical Society Conference on High Energy Physics

5-12 July, 2017

Venice

\section{${ }^{1}$ Speaker}




\section{1. Introduction}

The future Compact Linear Collider (CLIC) offers excellent research potential for precision measurements in the QCD-background-free environment of $\mathrm{e}^{+} \mathrm{e}^{-}$collisions. An energy-staged approach that includes three running center-of-mass energies $(350 \mathrm{GeV}, 1.4 \mathrm{TeV} \text { and } 3 \mathrm{TeV})^{2}$ maximizes the potential to explore the Higgs sector in a model-independent way using the favorable properties of Higgstrahlung, a Higgs production mechanism exploitable at future lepton colliders at center-of-mass energies $\leq 1 \mathrm{TeV}$. In addition, CLIC offers excellent possibilities to study top physics, including a threshold scan at the lowest center-of-mass energy, as well as to study the Higgs self-coupling and to perform Beyond Standard Model (BSM) searches at the highest energy.

\section{2.Higgs physics at CLIC}

Higgsstrahlung and WW-fusion are the dominant production mechanisms of the Higgs boson at low and high energies respectively. Combined they lead to $\sim 10^{6}$ Higgs bosons. Depending on the production mechanism, appropriate polarization could eventually double the statistics. Combined study of the two production mechanisms probe the Higgs width and couplings in a model-independent way. This leads to a determination of the Higgs couplings at the level of a percent (except for the rare decays to light particles such as muons or photons), employing all three stages. Assuming that the Higgs total width is constrained by the SM decays, the statistical precision of the Higgs couplings can be improved to a sub-percent level.

The expected number of Higgs bosons produced per energy stage, with unpolarized beams, is given in Table 1 [1], while Figure 1 illustrates the cross-section dependence on the available center-of-mass energies for various Higgs production mechanisms.

Higgsstrahlung events at $\sqrt{s}=350 \mathrm{GeV}$ allow us to perform a model independent measurement of the $\mathrm{ZH}$ coupling at CLIC, using the recoil mass technique. This measurement is unique to lepton colliders, and is possible due to the well-defined initial state and clean experimental conditions. By reconstructing only the $\mathrm{Z}$ decay products and calculating the mass of the system recoiling against it, the Higgs boson can be identified independently of its own decay. Leptonic $\mathrm{Z}$ decays give the cleanest signature, in these channels the $\mathrm{ZH}$ coupling can be measured with a statistical accuracy of $2 \%$. Hadronic $\mathrm{Z}$ decays can also be used, and large statistics of this channel give an uncertainty of $0.9 \%$, resulting in a combined uncertainty on the $\mathrm{ZH}$ coupling of $0.8 \%$.

\footnotetext{
${ }^{2}$ Different staging scenario for CLIC, with a first stage at $\sqrt{ } s=380 \mathrm{GeV}$ and the next stage at $1.5 \mathrm{TeV}$, has recently been adopted and will be used for future studies.
} 


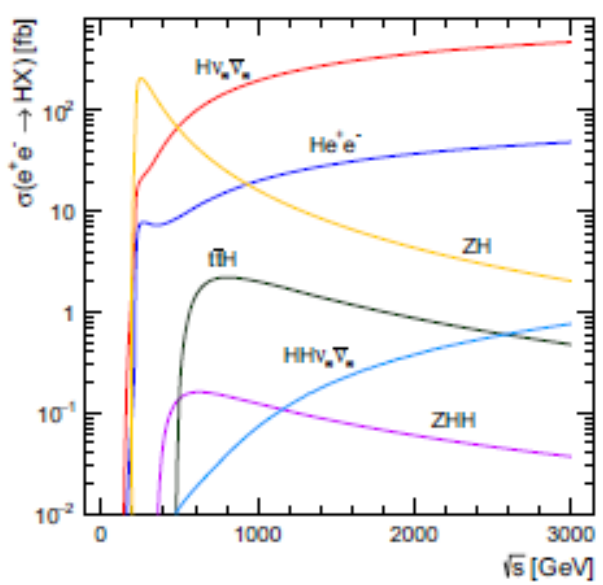

Figure 1. The cross section of various Higgs production processes as a function of centre-ofmass energy.

\begin{tabular}{lccc}
\hline$\sqrt{s}=$ & $350 \mathrm{GeV}$ & $1.4 \mathrm{TeV}$ & $3 \mathrm{TeV}$ \\
\hline $\mathcal{L}_{\text {int }}$ & $500 \mathrm{fb}^{-1}$ & $1500 \mathrm{fb}^{-1}$ & $2000 \mathrm{fb}^{-1}$ \\
$\sigma\left(e^{+} e^{-} \rightarrow Z H\right)$ & $133 \mathrm{fb}$ & $8 \mathrm{fb}$ & $2 \mathrm{fb}$ \\
$\sigma\left(e^{+} e^{-} \rightarrow H v_{e} \bar{v}_{e}\right)$ & $34 \mathrm{fb}$ & $276 \mathrm{fb}$ & $477 \mathrm{fb}$ \\
$\sigma\left(e^{+} e^{-} \rightarrow H e^{+} e^{-}\right)$ & $7 \mathrm{fb}$ & $28 \mathrm{fb}$ & $48 \mathrm{fb}$ \\
\#ZH events & 68000 & 20000 & 11000 \\
\# $v_{\mathrm{e}} \bar{v}_{\mathrm{e}}$ events & 17000 & 370000 & 830000 \\
\# $\mathrm{H} v_{\mathrm{e}} \bar{v}_{\mathrm{e}}$ events & 3700 & 37000 & 84000
\end{tabular}

Table 1. Assumed integrated luminosities, the leading-order unpolarized Higgs production crosssections and the expected numbers of events for the Higgsstrahlung, WW-fusion and ZZ-fusion processes, for simulated Higgs mass of $126 \mathrm{GeV}$, at the three centre-of-mass energies. The presented cross sections include the effects of ISR but exclude the effects of beamstrahlung. Also given are numbers of expected events, including the effects of ISR and the CLIC beamstrahlung spectrum. The presented cross sections and event numbers do not include possible enhancements from polarised beams.

Cross section times branching fraction measurements for the Higgs decay modes can be performed at the first energy stage of CLIC, with statistical precisions of a few \%. This includes 
the separation and measurement of the $\mathrm{H} \rightarrow \mathrm{bb}, \mathrm{H} \rightarrow \mathrm{cc}$ and $\mathrm{H} \rightarrow \mathrm{gg}$ decays. The invisible Higgs decay branching fraction can be constrained to $<1 \%$. At the higher energy stages, increasing cross sections and luminosity lead to higher statistics. The full CLIC programme will allow to access rare decays such as $\mathrm{H} \rightarrow \mu^{+} \mu^{-}, \mathrm{H} \rightarrow \gamma \gamma$ and $\mathrm{H} \rightarrow \mathrm{Z} \gamma$ with a statistical precision of $25 \%$ (at $3 \mathrm{TeV}$ ), $15 \%$ (at $1.4 \mathrm{TeV}$ ) and $42 \%$ (at $1.4 \mathrm{TeV}$ ), respectively.

At $\sqrt{s}=1.4 \mathrm{TeV}$, ttH production provides direct sensitivity to the top Yukawa coupling. With electron polarization of $-80 \%$, it is possible to achieve a relative statistical uncertainty of the top Yukawa coupling of $4.2 \%$ [1].

At the highest energy stage of CLIC double Higgs production is accessible in the HHvv state, enabling measurements of the Higgs self-coupling $\lambda$ and the quartic HHWW coupling. High energy and high luminosity, which give $1200 \mathrm{HHvv}$ events in $2 \mathrm{ab}^{-1}$ at $\sqrt{s}=3 \mathrm{TeV}$, are crucial for these analyses,. By reconstructing a four-jet topology the quartic coupling can be measured to $20 \%$. The Higgs self-coupling can be measured to $22 \%$ including electron beam polarisation.

To estimate the ultimate precision on the Higgs couplings and decay width at CLIC, results from all three energy stages are combined using a simultaneous fit. Two types of fits are used: A model-independent fit making minimal theoretical assumptions, and a model dependent fit following the strategies used for the interpretation of LHC Higgs results. In the Figure 2, the model-independent (model-dependent) couplings are shown to the left (right).
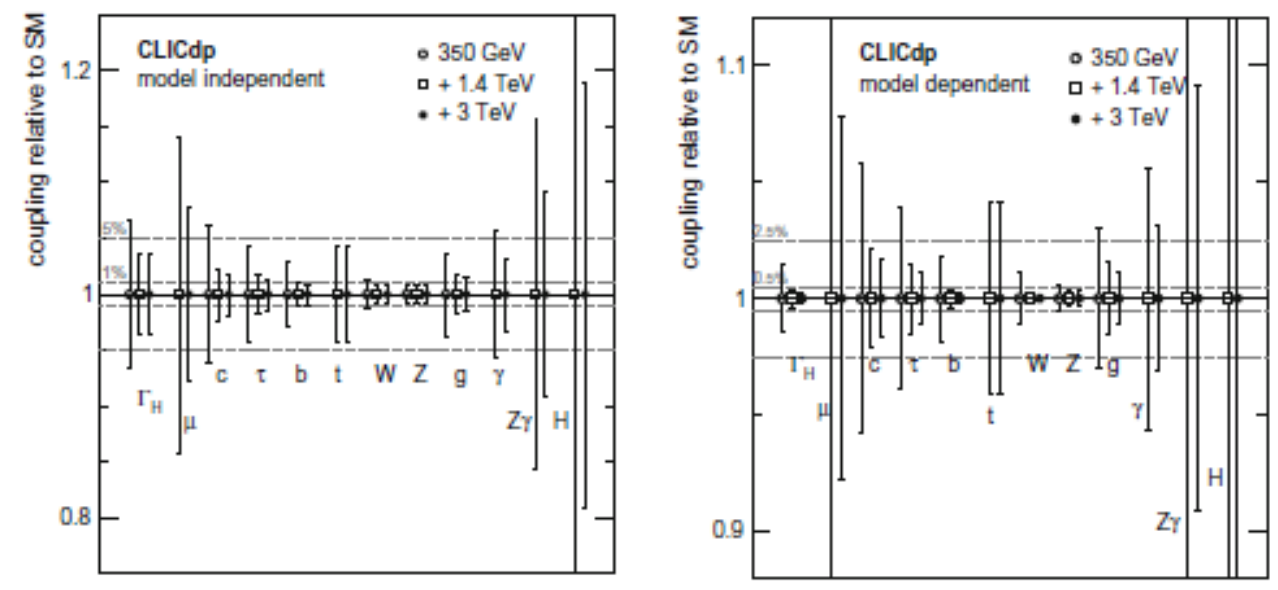

Figure 2. Illustration of the precision of the Higgs couplings and decay width of the studied threestage CLIC programme [1]. Left: Results using a model-independent fit. Right: Results using a model dependent fit.

\section{3.Beyond the Standard Model physics physics at CLIC}


At CLIC, potential signatures of BSM physics can be searched for either through direct reconstruction of new particles, with an approximate mass reach of $\sqrt{s} / 2$ (assuming pair production), and indirectly, in a model-dependent way, searcing for possible deviations in precision observables (like cross-sections, FB and LR asymmetries, etc.). Indirect searches extend the sensitivity for BSM physics beyond the direct kinematic reach of the machine.

Direct signal of supersymmetry can be determined at a percent level, assuming range of masses of $\approx 350 \mathrm{GeV}$ for the lightest neutralino, $\approx 480-650 \mathrm{GeV}$ for heavier neutralinos (charginos), approximately $550 \mathrm{GeV}-1.1 \mathrm{TeV}$ for charged sleptons, and $\approx 1.1 \mathrm{TeV}$ for lightflavored squarks, depending on a SUSY model (Model I, II, III [2]). Final state particles energy spectrum, such as $\mathrm{W}$ bosons or muons from $\mathrm{e}^{+} \mathrm{e}^{-} \rightarrow \tilde{\chi}_{1}^{+} \tilde{\chi}_{1}^{-} \rightarrow \mathrm{W}^{+} \mathrm{W}^{-} \tilde{\chi}_{1}^{0} \tilde{\chi}_{1}^{0}$ or $\mathrm{e}^{+} \mathrm{e}^{-} \rightarrow \tilde{\mu}_{R}^{+} \tilde{\mu}_{R}^{-} \rightarrow$ $\mu^{+} \mu^{-} \tilde{\chi}_{1}^{0} \tilde{\chi}_{1}^{0}$ can be used to measure masses of $\tilde{\chi}_{1}^{ \pm}$or $\tilde{\mu}_{1}^{+}$, as well as of neutralino (LSP). Energy distribution as shown in Figure 3 left [3], is distorted by the luminosity spectrum giving the systematic contribution to the uncertainty of masses of about $40 \mathrm{MeV}$. For a smuon mass around $1 \mathrm{TeV}$ and a $340 \mathrm{GeV}$ neutralino mass, the statistical uncertainty in mass determination is of the order of 5-6 GeV at $3 \mathrm{TeV}$ CLIC.

Very good jet energy resolution is crucial for proper reconstruction of gaugino decays to Higgs, $\mathrm{W}$ or $\mathrm{Z}$ bosons enabling statistical precision at a percent level or better, achievable at 3 $\mathrm{TeV}$ CLIC . Illustration of gaugino event reconstruction for three possible decay channels is given in Figure 3 right [4].
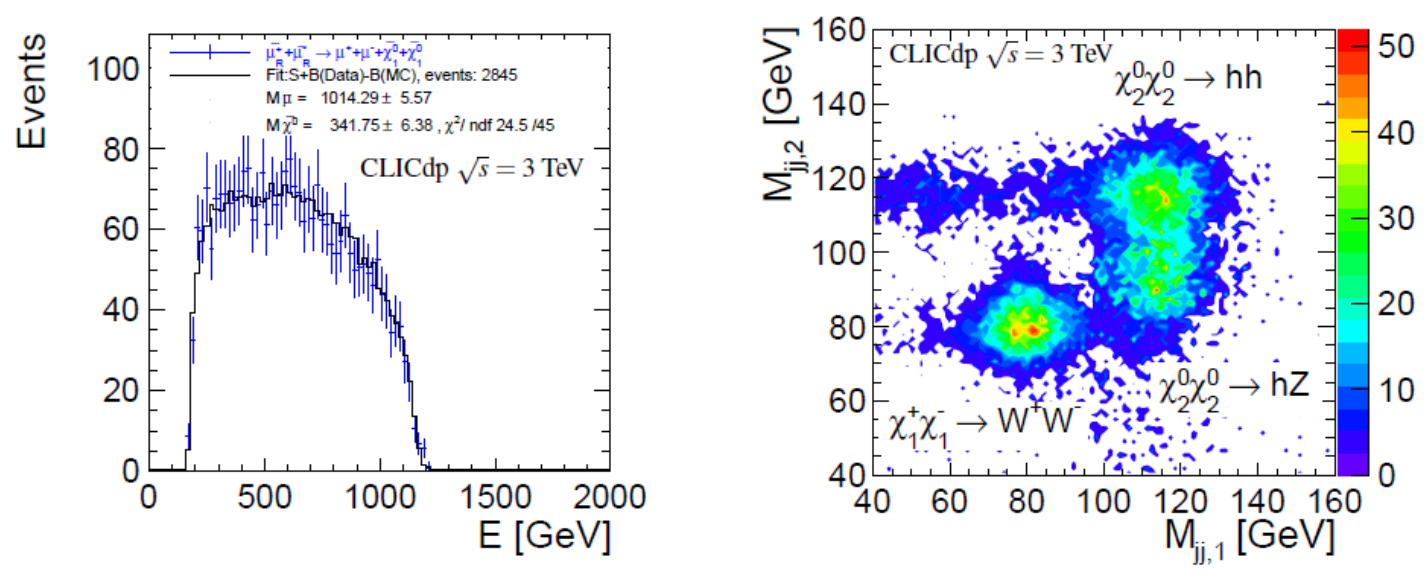

Figure 3. Left: The reconstructed energy of the di-muon pair in the decay $\mathrm{e}^{+} \mathrm{e}^{-} \rightarrow \tilde{\mu}_{R}^{+} \tilde{\mu}_{R}^{-} \rightarrow \mu^{+} \mu^{-}$ $\tilde{\chi}_{1}^{0} \tilde{\chi}_{1}^{0}$. The end-points of this spectrum give the slepton masses. Right: The reconstructed di-jet masses from chargino and neutralino decays.

In indirect searches, fermion-pair production can be used to test extended gauge theories (i.e. with Z') where sensitive observables receive higher order corrections due to the appearance of new gauge bosons. The sensitivity reaches masses of several tens of $\mathrm{TeV}$ (depending on the coupling assumptions), what is well beyond the available center-of-mass energy.

\section{4.Summary}


CLIC opens up the possibility of studying $\mathrm{e}^{+} \mathrm{e}^{-}$collisions with $\sqrt{s}>1 \mathrm{TeV}$. The clean environment and the low level of background provided by lepton collisions combined with the high centre-of-mass energy gives a great potential for both SM physics and BSM searches. At the first stage of $350 \mathrm{GeV}$ a model independent measurement of the $\mathrm{ZH}$ coupling can be performed. Higher energy stages, up to $3 \mathrm{TeV}$, improve statistics and give access to rare Higgs decays, the top Yukawa coupling and the Higgs self-coupling. Results from the Higgs studies can be combined in a model independent and model dependent approach, resulting in a sub-percent coupling precision observables. SUSY particle masses could be directly measured up to the kinematic limit. Indirect searches increase the reach of CLIC up to tens of TeV.

\section{Acknowledgments}

The Vinca Institute activity at CLIC/CLICdp has been supported by the Ministry of Education, Science and Technological Development of the Republic of Serbia, through the project OI171012.

\section{References}

[1] H. Abramowicz et al., Higgs Physics at the CLIC Electron-Positron Linear Collider, EPJ C 77, 475, 2017.

[2] L. Linssen et al. (editors), Physics and Detectors at CLIC: CLIC Conceptual Design Report, CERN2012-003, CERN, 2012.

[3] M. Battaglia, J-J. Blaising, J. S. Marshall, S. Poss, M. Thomson, A. Sailer, E. van der Kraaij, Physics performance for Scalar Electron, Scalar Muon and Scalar Neutrino searches at $\sqrt{ } s=3 \mathrm{TeV}$ and $1.4 \mathrm{TeV}$ at CLIC, JHEP 1309 (2013) 001.

[4] T. Barklow, A. Munnich and P. Roloff, Measurement of chargino and neutralino pair production at CLIC, 2011, CERN LCD-Note-2011-037. 